\title{
Magnetic resonance spectroscopy in pediatric brain tumors: how to make a more confident diagnosis
}

\author{
Noha M. Attia ${ }^{1 *} \mathbb{D}$, Samy Abdel Aziz Sayed', Khaled Fathy Riad² and Ghada Mahmoud Korany ${ }^{1}$
}

\begin{abstract}
Background: Non-invasive diagnosis of pediatric brain tumors can be challenging due to diverse tumor pathologies and similar imaging appearances. Magnetic resonance spectroscopy (MRS), when combined with high spatial resolution anatomic imaging obtained with conventional magnetic resonance imaging (MRI), provides metabolic information within the lesion as well as the surrounding tissue. The differentiation of neoplastic from non-neoplastic lesions and low-grade from high-grade neoplasms is essential for determining the choice of treatment and the best treatment plan. We aimed to measure specific metabolic ratios and evaluate metabolic profiles of various lesions by MRS to assist in making a more confident diagnosis.

Results: The choline/creatine (Cho/Cr), choline/N-acetylaspartate (Cho/NAA), and Cho/NAA+Cr ratios all had statistically significant values for the differentiation between neoplastic and non-neoplastic lesions at cutoffs 1.8, 2, and 0.8 respectively. The Cho/NAA, Cho/Cr, Cho/NAA+Cr, and myo-inositol/creatine $(\mathrm{ml} / \mathrm{Cr}$ ) ratios all had statistically significant values for the differentiation of high-grade from low-grade neoplasms at cutoffs 3.3, 3.5, 1.3, and 1.5 respectively. The presence of a lipid lactate peak was only significant for differentiating high-grade from low-grade neoplasms. Medulloblastomas, diffuse pontine gliomas, and choroid plexus carcinoma all showed characteristic metabolic profiles on MRS. Metastasis showed lower Cho/ NAA and Cho/Cr ratios outside the tumor margin than high-grade neoplasms.

Conclusion: The use of certain metabolite ratios with high sensitivity and specificity to distinguish neoplastic from nonneoplastic lesions and low-grade from high-grade neoplasms while assessing the metabolic profile of the lesion aids in the non-invasive diagnosis of pediatric brain tumors. MRS facilitates earlier treatment planning by determining tumor spatial extent and predicting tumor behavior with potential to solve sampling problems of inaccessible and heterogenous lesions as well as unnecessary sampling of benign lesions.
\end{abstract}

Keywords: Brain, Tumors, Pediatric, MR spectroscopy, Metabolic ratios

\section{Background}

Magnetic resonance imaging (MRI) offers a non-invasive approach for diagnosis, grading, and post-treatment follow-up of pediatric brain tumors. Although it is considered the gold standard for the evaluation of brain tumors, in many instances, reliable differentiation of neoplastic from non-neoplastic brain lesions or of high from low-grade tumors is difficult with conventional MRI [1]. Several types of non-neoplastic brain lesions (abscess, vasculitis, etc.) can be misdiagnosed as brain

\footnotetext{
* Correspondence: nohamohamedali@yahoo.com

'Radiology Department, Faculty of Medicine, Assiut University Hospitals,

Assiut University, Assiut 71526, Egypt

Full list of author information is available at the end of the article
}

tumors. As a result, unnecessary brain biopsies may be performed on patients with benign lesions. Differentiation of high from low-grade tumors can be challenging due to diverse tumor pathologies as well as nonspecific or similar imaging findings. Therefore, accurate diagnosis is important in influencing the choice of treatment and determining the best treatment plan [2].

MR spectroscopy (MRS) can assess pediatric neurological abnormalities at microscopic levels by quantifying cellular metabolites and studying their distribution in the tissues. The most widely available MRS method is proton $\left({ }^{1} \mathrm{H}\right.$; hydrogen) spectroscopy. MRS has been proven to provide additional clinically relevant information for several pediatric neurological disease processes 
such as brain tumors, infectious processes, white matter disorders, and neonatal injury [3]. When combined with high resolution anatomical imaging provided by conventional MRI, MRS biomarkers can complement neuropathological data and enhance insight into therapeutic options [4]. Although MRS is becoming widely available, few studies have evaluated how it can enhance conventional radiological reporting by adding value to information obtained through MRI alone [5]. This study aims to evaluate certain MRS metabolic ratios and metabolic profiles to assist in making a more confident diagnosis of pediatric brain tumors.

\section{Methods}

\section{Patients}

Thirty patients (16 females and 14 males) in the pediatric age group ranging between 1 and 16 years with mean age $( \pm$ SD) of $11.56 \pm 2.48$ years were prospectively recruited for this study. They presented to the pediatric oncology clinic with cerebral mass lesions diagnosed by $\mathrm{CT}$ in the period between May 2016 and May 2018. Informed written consents were obtained from the patients' guardians according to the National Ethics Committee. All patients were subjected to thorough clinical examination, conventional magnetic resonance imaging, and magnetic resonance spectroscopic study using 1.5T scanner (Achieva, Philips Healthcare; Amsterdam, Netherlands) equipped with the standard head coil. Some cases were excluded from the study due to masses that were too close to the calvarium or paranasal sinuses which resulted in a poor quality diagnostic spectrum.

\section{Conventional MRI technique}

Axial and sagittal pre-contrast T1-weighted spin echo, axial FLAIR, and axial and coronal T2-weighted fast spin-echo images were performed. Then, contrast-enhanced axial, sagittal, and coronal T1-weighted spin-echo images using Gad-DTPA (Magnevist or Omniscan) $0.1 \mathrm{mmol} / \mathrm{kg}$ were also performed.

\section{MRS technique}

Point Resolved Spatial Selection (PRESS) was done at intermediate echo time (TE) $144 \mathrm{~ms}$. The voxel size inside spectroscopic grid was $10 \times 10 \mathrm{~mm}$. The spectroscopic grid was extended and manually adjusted to include lesion, perilesional edema if present, and normal brain tissue. The volume of interest (VOI) was compared with the contralateral hemisphere having a normal MRI appearance. The scan time was approximately $5 \mathrm{~min}$. 2D short TE sequence at $35 \mathrm{~ms}$ was also used. The scan time was approximately $12 \mathrm{~min}$.

The peak ratios were obtained by measuring and comparing areas under the curve (AUC). The peaks were assigned as follows: choline (Cho) peak at $3.2 \mathrm{ppm}$, creatine $(\mathrm{Cr})$ at $3 \mathrm{ppm}, \mathrm{N}$-acetylaspartate (NAA) peak at $2.02 \mathrm{ppm}$, mobile lipids at $0.5-1.5 \mathrm{ppm}$, lactate $1.44 \mathrm{ppm}$, and myo-inositol (mI) at $3.56 \mathrm{ppm}$.

\section{Image analysis}

Maximum high Cho/NAA, Cho/Cr, and $\mathrm{Cho} / \mathrm{NAA}+\mathrm{Cr}$ peak ratios were recorded in the lesion at intermediate TE $144 \mathrm{~ms}$ as well as minimum NAA/Cho and NAA/Cr ratios. The $\mathrm{ml} / \mathrm{Cr}$ ratio was recorded in the lesion at short TE $35 \mathrm{~ms}$. The Cho/NAA and Cho/Cr were recorded in the perilesional edema if present at intermediate TE $144 \mathrm{~ms}$. Lipid signal presence or absence was also recorded. The lesions were initially diagnosed as neoplastic or non-neoplastic and then the neoplastic group was subcategorized into low or high-grade tumors.

The MRI and MRS examinations were analyzed by two experienced radiologists (radiologist 1 with 7 years of experience and radiologist 2 with 23 years) blinded to the clinical information regarding presentation and to the laboratory tests and the demographic findings. All decisions were made by means of consensus. Twenty-six of the 30 cases underwent stereotactic biopsy. All specimens were histologically examined by a neuropathologist and graded according to the World Health Organization (WHO) classification. Histopathological results were compared with MRS results.

\section{Statistical analysis}

Statistical analysis was performed using the Statistical Package for the Social Sciences (SPSS version 18) software. The results were expressed as means \pm standard deviation while qualitative data were expressed as frequencies and percentages.

Receiver operating characteristic (ROC) curve analysis was used to correlate the histopathological results with the spectroscopic metabolite ratios. The area under the curve (AUC) was used to calculate the optimal cutoff values for differentiating neoplastic versus nonneoplastic lesions and low-grade versus high-grade neoplasms. $P$ values $<0.05$ were considered significant.

\section{Results}

Thirty MRI and MRS examinations were performed, and 28 of them were found to have intracranial mass lesions. The remaining two cases were diagnosed as follows: one case with multiple sclerosis and the other case with acute disseminated encephalomyelitis (ADEM) based on a clinical and radiological follow-up. Twenty-six of the 28 cases with mass lesions underwent stereotactic biopsy and were diagnosed histopathologically. Two cases with a brainstem glioma had no biopsy or surgery and were followed up clinically and radiologically. The distribution of these lesions is shown in Table 1.

The ROC curve analysis revealed that the $\mathrm{Cho} / \mathrm{Cr}$, $\mathrm{Cho} / \mathrm{NAA}$, and $\mathrm{Cho} / \mathrm{NAA}+\mathrm{Cr}$ all had statistically significant values for the differentiation between neoplastic and non-neoplastic lesions with variable sensitivities and specificities (Table 2). The Cho/NAA $+\mathrm{Cr}$ ratio had the 
Table 1 Distribution of final diagnosis among studied patients based on pathological diagnosis or clinical-laboratory diagnosis and follow-up imaging

\begin{tabular}{ll}
\hline Method of final diagnosis & $N=30$ \\
\hline Histopathology & $26(86.7 \%)$ \\
High-grade astrocytoma & $4(13.3 \%)$ \\
Low-grade astrocytoma & $7(23.3 \%)$ \\
Medulloblastoma & $2(6.7 \%)$ \\
Ependymoma & $2(6.7 \%)$ \\
Anaplastic ependymoma & $2(6.7 \%)$ \\
PXA & $2(6.7 \%)$ \\
Brain abscess & $2(6.7 \%)$ \\
Choroid plexus carcinoma & $1(3.3 \%)$ \\
Metastatic lesion & $1(3.3 \%)$ \\
Recurrence of GBM & $1(3.3 \%)$ \\
DNET & $1(3.3 \%)$ \\
PNET & $1(3.3 \%)$ \\
Clinical with laboratory diagnosis & $4(13.3 \%)$ \\
Brainstem glioma & $2(6.7 \%)$ \\
Multiple sclerosis & $1(3.3 \%)$ \\
ADEM & $1(3.3 \%)$ \\
Total & 30 \\
\hline
\end{tabular}

Data was expressed in the form of frequency (percentage). DENT dysembryoplastic neuroepithelial tumor, PENT primitive neuroectodermal tumor, PXA pleomorphic xanthoastrocytoma, ADEM acute disseminated encephalomyelitis

highest sensitivity and specificity for the prediction of neoplastic lesions equal to $88 \%$ and $100 \%$ respectively at a cutoff point $>0.8$ with $\mathrm{AUC}=0.91$ and $P$ value $=0.009$. The Cho/NAA ratio showed similar results at a cutoff point $>2$; it had $88 \%$ sensitivity and $75 \%$ specificity with AUC $=0.91$ and $P$ value $=0.01$. The $\mathrm{Cho} / \mathrm{Cr}$ ratio at a cutoff point $>1.86$ had $85 \%$ sensitivity and $75 \%$ specificity with $\mathrm{AUC}=0.85$ and $P$ value $=0.02$. However, the $\mathrm{NAA} / \mathrm{Cr}$ and myo-inositol/Cr ratios showed no statistically significant values for the prediction of neoplastic lesions with low AUC and insignificant $P$ values.

Regarding the prediction of high-grade versus low-grade lesions, the ROC curve analysis revealed that the Cho/ $\mathrm{NAA}, \mathrm{Cho} / \mathrm{Cr}$, Cho/NAA+Cr, and $\mathrm{mI} / \mathrm{Cr}$ ratios all had statistically significant results (Table 3 ). The analysis showed that the Cho/NAA at a cutoff point $>3.3$ had $92 \%$ sensitivity and $72 \%$ specificity with $\mathrm{AUC}=0.87$ and $P$ value $=0.001$ while the $\mathrm{Cho} / \mathrm{Cr}$ ratio at a cutoff point $>3.5$ had $83 \%$ sensitivity and $78 \%$ specificity with $\mathrm{AUC}=0.81$ and $P$ value $=0.005$. Furthermore, the Cho $/ \mathrm{NAA}+\mathrm{Cr}$ ratio at a cutoff point $<1.3$ had $83 \%$ sensitivity and $72 \%$ specificity with AUR $=0.85$ and $P=0.002$.

Myo-inositol was significantly elevated in low-grade lesions. The $\mathrm{mI} / \mathrm{Cr}$ ratio showed $83 \%$ sensitivity and $79 \%$ specificity for the prediction of high-grade lesions at a cutoff $<$
Table 2 ROC curve analysis of the various metabolite ratios for the prediction of neoplastic lesions

\begin{tabular}{lllll}
\hline & Cho/NAA & $\mathrm{Cho/Cr}$ & $\mathrm{Cho} / \mathrm{NAA}+\mathrm{Cr}$ & $\mathrm{NAA} / \mathrm{Cr}$ \\
\hline Sensitivity & $88 \%$ & $85 \%$ & $88 \%$ & - \\
Specificity & $75 \%$ & $75 \%$ & $100 \%$ & - \\
Optimal cutoff & $>2$ & $>1.86$ & $>0.8$ & - \\
AUC & 0.91 & 0.85 & 0.91 & 0.71 \\
$P$ value & 0.01 & 0.02 & 0.009 & 0.2 \\
\hline
\end{tabular}

$P$ value was significant if $<0.05, A U C$ area under the curve

1.5 with $\mathrm{AUC}=0.9$ and $P$ value $=0.001$. However, the $\mathrm{NAA} / \mathrm{Cr}$ ratio showed statistically insignificant results for the differentiation between low- and high-grade neoplasms.

The presence of a lipid lactate peak showed low sensitivity and specificity for the differentiation of neoplastic from non-neoplastic lesions. However, it was valuable for the differentiation of low-grade from high-grade lesions with a sensitivity of $75 \%$ and specificity of $72 \%$ and $P$ value $=0.03$.

Four types of tumors were visualized in the posterior fossa in our study which are medulloblastomas, pilocytic astrocytomas, DNET, and ependymomas. Two cases were histologically proven to be medulloblastomas. They showed higher levels of choline than the other posterior fossa tumors with slight increase of myo-inositol, low NAA levels, lactate peaks, and no lipids. They had $\mathrm{Cho} / \mathrm{Cr}$ ratio ranging from 10.6 to 14.8 with mean $12.7 \pm 7.51$, Cho/NAA ratio ranging from 22.5 to 26 with mean $24.53 \pm 7.03$, Cho/NAA+Cr ratio ranging from 5.5 to 7 with mean $6.37 \pm 0.78$, and NAA/Cho ratio ranging from 0.04 to 0.07 with mean $0.05 \pm 0.04$ (Figs. 1 and 2).

On the other hand, pilocytic astrocytomas and DNET showed just mild increased Cho peak and Cho/Cr ratio reflecting their low aggressiveness. Additionally, NAA is present at higher levels in comparison with other types of tumors, suggesting low levels of neuronal destruction.

Four cases of ependymomas were diagnosed; two of them were pathologically proven to be grade II ependymoma and two were grade III anaplastic ependymoma. The choline levels in both grades were intermediate falling between medulloblastoma and pilocytic astrocytomas. However, they were lower in grade II than in grade III ependymomas with $\mathrm{Cho} / \mathrm{Cr}$ ratio ranging from 2.77

Table 3 ROC curve analysis of the metabolite ratios for the prediction of high-grade neoplastic lesions

\begin{tabular}{lllll}
\hline & Cho/NAA & Cho/Cr & Cho/NAA+Cr & Myo-inositol (ml/Cr) \\
\hline Sensitivity & $92 \%$ & $83 \%$ & $83 \%$ & $83 \%$ \\
Specificity & $72 \%$ & $78 \%$ & $72 \%$ & $79 \%$ \\
Optimal cutoff & $>3.3$ & $>3.5$ & $>1.3$ & $<1.5$ \\
AUC & 0.87 & 0.81 & 0.85 & 0.9 \\
$P$ value & 0.001 & 0.005 & 0.002 & 0.001
\end{tabular}

$P$ value was significant if $<0.05$. AUC area under the curve 

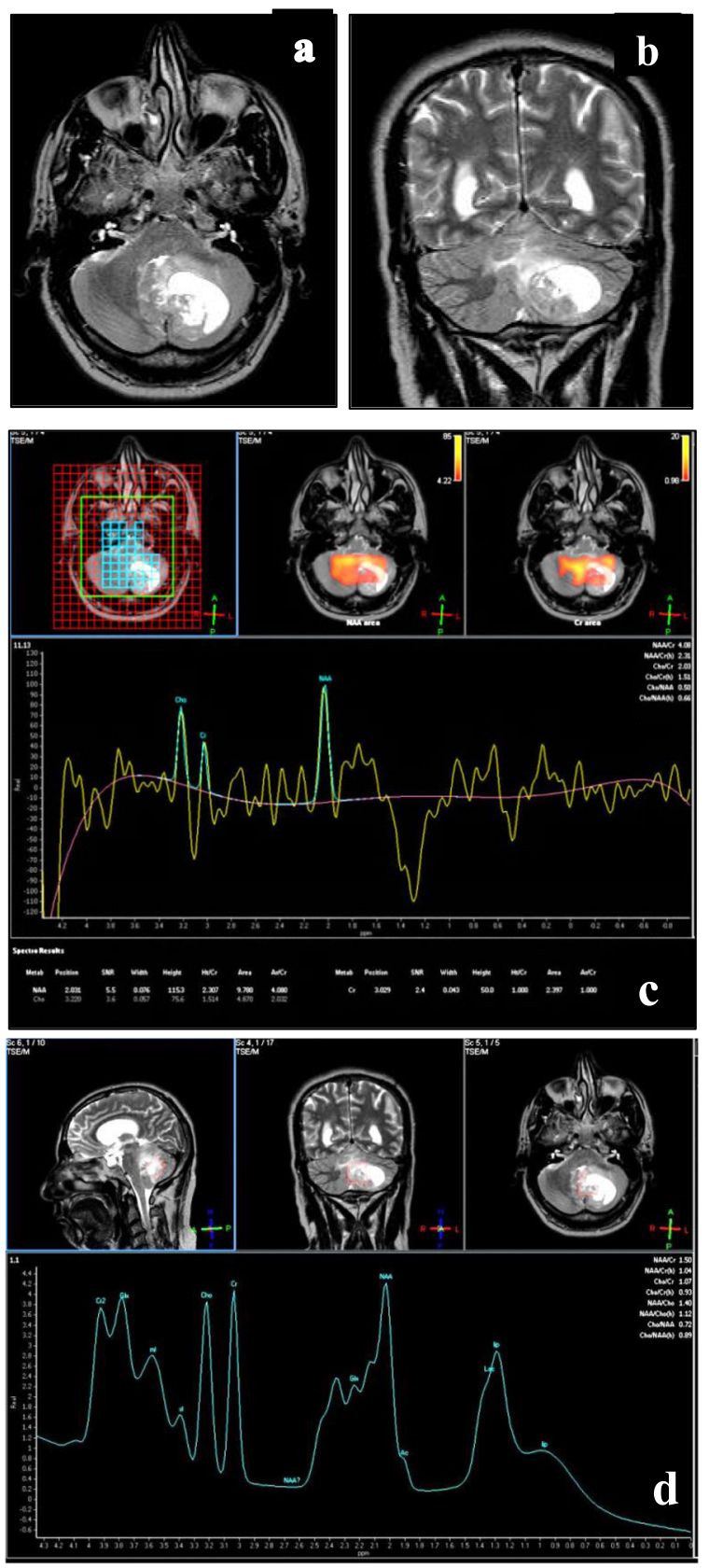

Fig. 1 A 12-year-old male patient presented with headache. a, b Axial and coronal T2WI show a heterogenous hyper intense left cerebellar and vermian SOL with mixed solid and cystic components. c 2D PRESS 144 ms echo spectrum shows mild elevation of choline peak and nearly normal $\mathrm{Cr}$ and NAA. The Cho/ $\mathrm{Cr}$ ratio $=2, \mathrm{Cho} / \mathrm{NAA}$ ratio $=0.5, \mathrm{Cho} / \mathrm{NAA}+\mathrm{Cr}$ ratio $=1.2, \mathrm{NAA} / \mathrm{Cho}$ ratio $=2$, and $\mathrm{NAA} / \mathrm{Cr}$ ratio $=4.1$ with inverted lactate peak. $\mathbf{d} 2 \mathrm{D}$ short TE 26 ms echo spectrum shows additional equivocal myoinositol peak $(\mathrm{ml} / \mathrm{Cr}$ ratio $=0.72)$ and a lipid peak. MRS diagnosis: primary low-grade astrocytoma. Histopathologically proved: WHO grade I cystic astrocytoma to 2.9 in low grade and from 15.8 to 25 in high grade while the Cho/NAA ratio ranging from 4.43 to 5.6 in low grade and from 11.3 to 20 in high grade. The myoinositol level was higher in both ependymoma grades than in medulloblastomas and pilocytic astrocytomas. However, both ependymomas and medulloblastomas had lactate peaks with no lipids.

The two cases of diffuse pontine gliomas in our study were diagnosed radiologically and by clinical follow-up. They showed elevation of choline, reduced NAA, and creatine with $\mathrm{Cho} / \mathrm{Cr}$ ratio $=2$ and 1.5 , Cho/NAA ratio $=$ 1.9 and 3 , as well as $\mathrm{Cho} / \mathrm{NAA}+\mathrm{Cr}$ ratio $=0.9$ and 1 . They showed no lipids or lactate.

A case was diagnosed with choroid plexus carcinomas which are usually cellular hyperperfused tumors. On MRS, it showed a very prominent choline peak with low NAA, Cr, and myo-inositol with $\mathrm{Cho} / \mathrm{Cr}$ ratio $=17$, Cho/ $\mathrm{NAA}$ ratio $=6$, and $\mathrm{Cho} / \mathrm{NAA}+\mathrm{Cr}$ ratio $=4$. A lipid $/$ lactate peak was also present suggesting its malignant nature.

Spectroscopic ratios of metastases from rhabdomyosarcoma (only one case) measured outside the tumor margin showed lower Cho/NAA ratio (0.5) and lower $\mathrm{Cho} / \mathrm{Cr}$ ratio (0.7). In high-grade gliomas, spectroscopic ratios outside the tumor margin showed higher Cho/NAA ratio ranging from 1.7 to 7 with mean $3.23 \pm 2.42$ and higher $\mathrm{Cho} / \mathrm{Cr}$ ratio ranging from 2.4 to 3.5 with mean $2.53 \pm 1.38$.

Two cases of cerebral abscesses showed significant amino acid peak at $0.9 \mathrm{ppm}$, lactate peak at $1.4 \mathrm{ppm}$, acetate peak $1.9 \mathrm{ppm}$, pyruvate/succinate not assigned peak at 2.4 with no elevation of Cho, and dwarfing of other metabolites, and such spectrum is specific for brain abscess (Fig. 3).

The spectroscopic findings of the case diagnosed with MS was not characteristic showing mildly reduced NAA levels in the lesions and in the adjacent white matter as well as slightly elevated myo-inositol peak. On diffusion weighted imaging (DWI), the MS lesions showed elevated ADC values. There was a single case of ADEM in our study which showed reduced NAA level, elevated choline, and myo-inositol peaks similar to low-grade gliomas. However, the discriminating factor was the presence of a lactate peak. On DWI, it showed reduced ADC values.

\section{Discussion}

Brain tumors are the most prevalent type of solid cancer in childhood, are the most common cause of pediatric cancer death, and are a significant cause of long-term disability [6]. Histopathology is still the gold standard in diagnosing pediatric brain tumors; however, even image-guided biopsies have an appreciable morbidity and mortality. Additionally, the heterogeneity of some tumors introduces problems with "sampling" error and undergrading of the tumor [7].

Noninvasive and accurate differentiation between neoplastic and non-neoplastic pediatric brain lesions as well 

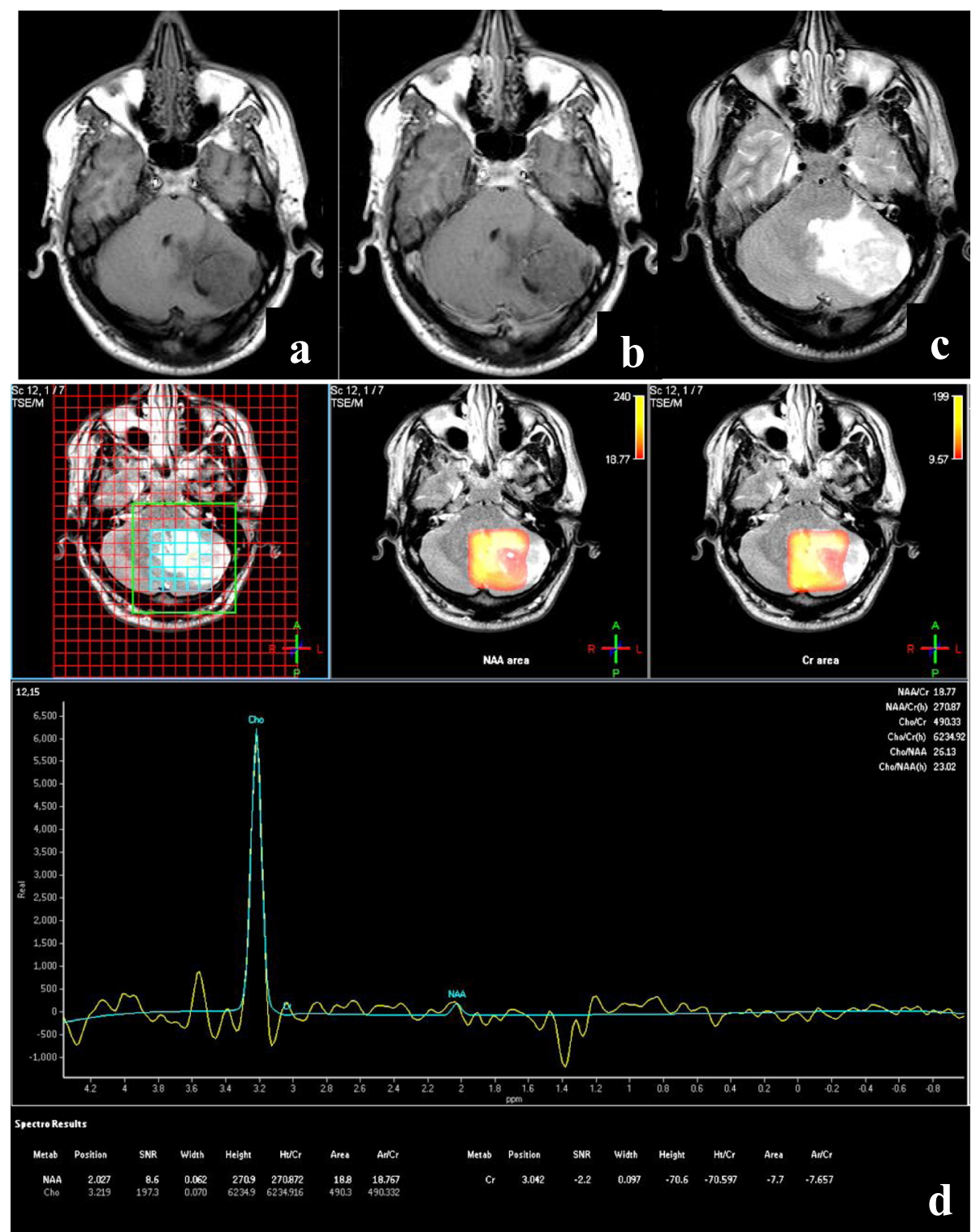

Fig. 2 A 16-year-old male patient presented with ataxic gait. a, b Axial T1WI pre- and post-contrast shows a hypointense SOL in the left cerebellar hemisphere acquiring minimal enhancement after contrast administration. c On axial T2WI, it shows heterogenous hyper intense signal. d 2D PRESS $144 \mathrm{~ms}$ echo spectrum shows remarkable elevation of choline peak with marked reduction of NAA and near absence of creatine. The $\mathrm{Cho} / \mathrm{Cr}$ ratio $=14.8$, the Cho/NAA ratio $=13.4$, the $\mathrm{Cho} / \mathrm{NAA}+\mathrm{Cr}$ ratio $=7, \mathrm{NAA} / \mathrm{Cho}$ ratio $=0.07$, and NAA/Cr ratio $=1.1$. MRS diagnosis in favor of primary high-grade malignant lesion; likely medulloblastoma. Histopathologically proved: medulloblastoma

as low- and high-grade neoplasms is challenging and important in determining the correct treatment plan. MRS provides information about biochemical characteristics, metabolic heterogeneity of the lesion, and the surrounding brain tissue [8].

Previous studies have shown the ability of various metabolite ratios to diagnose different brain lesions with varying sensitivities and specificities. Most of the studies used Cho/NAA, Cho/Cr, and NAA/Cho ratios; however, relying on these ratios alone proved to have certain fallacies [9]. In order to improve the diagnostic accuracy of
MRS, the current study also evaluated the $\mathrm{Cho} / \mathrm{Naa}+\mathrm{Cr}$ and the myo-inositol/Cr ratios.

In this study, the Cho/NAA $+\mathrm{Cr}$ and Cho/NAA ratios had the highest sensitivity and specificity for differentiation of neoplastic from non-neoplastic lesions. Using ROC curve analysis, the Cho/NAA+Cr had $88 \%$ sensitivity and $100 \%$ specificity for the prediction of neoplastic lesions at a cutoff point $>0.8$ with $\mathrm{AUC}=0.91$ and $P$ value $=0.009$. The Cho/NAA ratio showed similar results at a cutoff point $>2$ having $88 \%$ sensitivity and $75 \%$ specificity with $\mathrm{AUC}=0.91$ and $P$ value $=0.01$. The Cho/ 

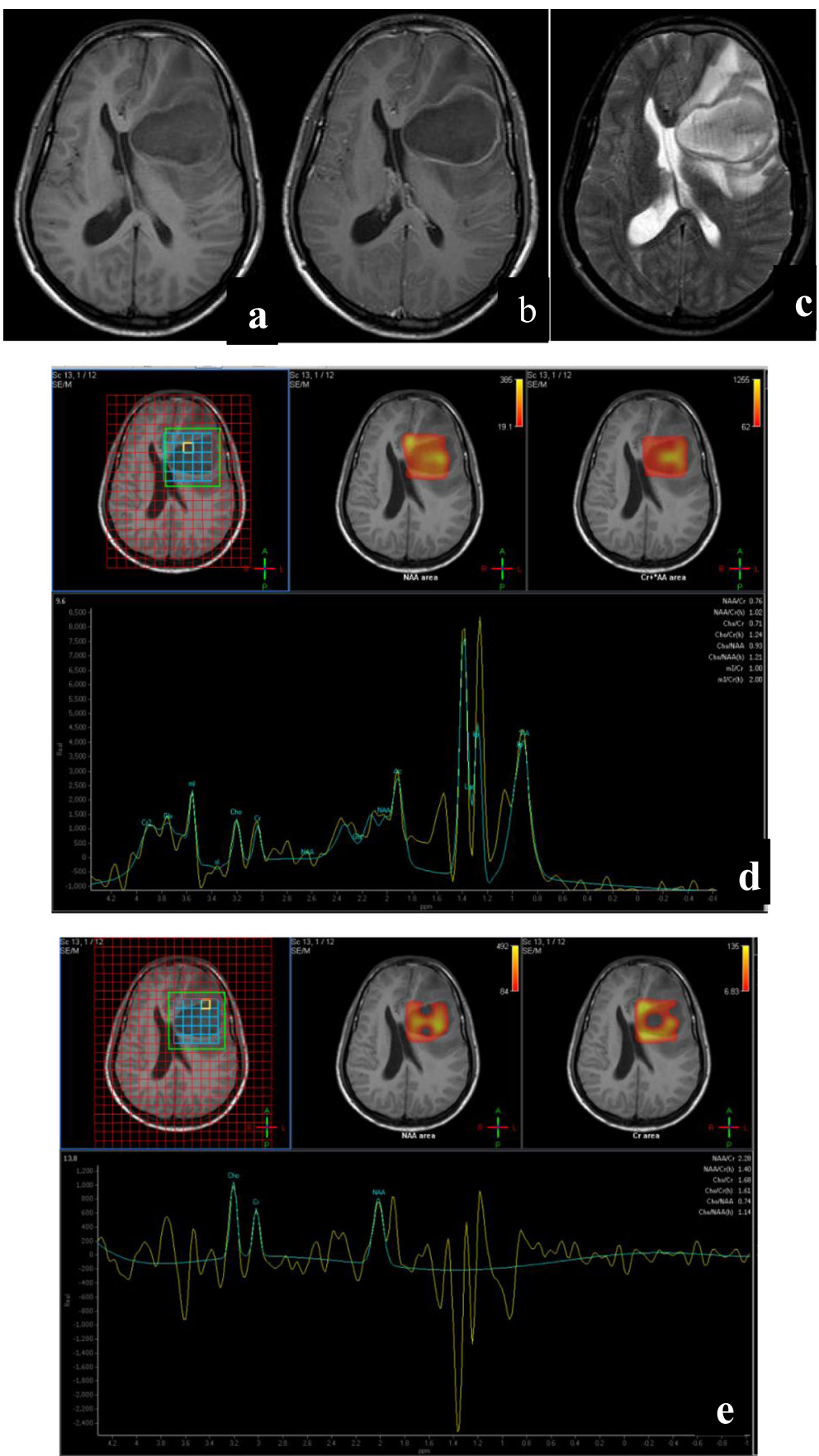

Fig. 3 An 11-year-old male presented with a fever and disturbed conscious level. a-c A left frontotemporal thin-walled cystic lesion; the wall is mildly hyperintense on T1 and hypointense on T2 with moderate peripheral enhancement. $\mathbf{d} 2 \mathrm{D}$ short TE 26 ms echo spectrum in the lesion shows significant amino acid peak at 0.9 ppm, lactate peak at 1.4 ppm, acetate peak 1.9 ppm, pyruvate/succinate not assigned peak at 2.4 with no elevation of Cho, and dwarfing of other metabolites. e 2D PRESS intermediate TE $144 \mathrm{~ms}$ shows maximum metabolite ratios, $\mathrm{Cho} / \mathrm{Cr}=1.7, \mathrm{Cho} /$ $\mathrm{NAA}=1, \mathrm{Cho} / \mathrm{NAA}+\mathrm{Cr}=0.7$, minimal NAA/Cho $=1, \mathrm{NAA} / \mathrm{Cr}=1.5$. The conventional and MRS features are suggestive of pyogenic brain abscess; pathologically proven

Cr ratio at a cutoff point $>1.86$ had $85 \%$ sensitivity and $75 \%$ specificity with $\mathrm{AUC}=0.85$ and $P$ value $=0.02$.

These results were found to be in agreement with the results of Karatag et al. [10], who reported that a Cho/
NAA ratio > 1.83 showed $87.2 \%$ sensitivity and $100 \%$ specificity while a Cho/Cr ratio $>1.98$ showed $71.8 \%$ sensitivity and $100 \%$ specificity for differentiation of neoplastic from non-neoplastic lesions. McKnight et al. [11] 
also reported that Cho/NAA ratio correlates with cell density and cell proliferation index. They also found that a ratio greater than 2 shows $96 \%$ sensitivity and $70 \%$ specificity for differentiating neoplastic from nonneoplastic lesion. Butzen et al. [12], however, reported a lower cutoff value of the Cho/NAA ratio being $>1$ to indicate a neoplastic process with a sensitivity of $79 \%$ and specificity of $77 \%$. On the other hand, the NAA/Cr ratio in this study showed no statistically significant values for the prediction of neoplastic lesions with low AUC as well as sensitivity and specificity.

The radiologic grading of gliomas using conventional MRI has a sensitivity ranging from 55 to $83 \%$ due to similar imaging characteristics of some low-grade and high-grade neoplasms. Attempts to grade tumors using MRS suggest that with increasing grade, there is an increase in the Cho/Cr ratio, a reduction in NAA, and increase in lactate/lipid peak [13]. Kapsalaki et al. [14] also demonstrated that the higher the Cho/NAA ratio is, the higher the astrocytoma grade.

In this study, the ROC curve analysis showed that the Cho/NAA at a cutoff point $>3.3$ had $92 \%$ sensitivity and $72 \%$ specificity for the prediction of high-grade neoplasms while the $\mathrm{Cho} / \mathrm{Cr}$ ratio at a cutoff point $>3.5$ had $83 \%$ sensitivity and $78 \%$ specificity. Furthermore, the Cho/ $\mathrm{NAA}+\mathrm{Cr}$ ratio at a cutoff point $>1.3$ had $83 \%$ sensitivity and $72 \%$ specificity and the NAA/Cho ratio showed $92 \%$ sensitivity and $95 \%$ specificity at a cutoff of $<0.5$.

This was in concordance with the results of Karatag et al. [10], who reported that a Cho/NAA ratio $>3.2$ shows sensitivity of $82.6 \%$ and specificity of $100 \%$ while a Cho/Cr ratio $>2.2$ shows $95.7 \%$ sensitivity and $84.6 \%$ specificity in differentiation of low-grade versus high-grade neoplastic lesions. Oz et al. [15] also reported that Cho/NAA ratio greater than 2, a Lac/NAA ratio greater than 0.25 , and the presence of lipid at MR spectroscopic imaging are characteristics of a high-grade tumor.

Myo-inositol is one of the most abundant metabolites visible on MRS at short TE. It is involved in the production of proteolytic enzymes found in aggressive primary tumors through activation of protein $C$ [16]. Consequently, the $\mathrm{MI} / \mathrm{Cr}$ ratio was measured in this study to help improve the prediction of tumor grade by MRS. The level of myo-inositol was significantly higher in low-grade gliomas compared with high-grade gliomas. At a cutoff point $<1.5$, the $\mathrm{MI} / \mathrm{Cr}$ ratio had $83 \%$ sensitivity and $79 \%$ specificity for prediction of high-grade neoplastic lesions.

These results were similar to those of Castillo et al. [17], who compared $\mathrm{mI} / \mathrm{Cr}$ ratio in 20 patients with high-grade glioma, 14 patients with low-grade glioma, and 5 control cases. Castillo found elevated $\mathrm{mI} / \mathrm{Cr}$ in low-grade glioma with mean $\mathrm{mI} / \mathrm{Cr} 0.8 \pm 0.25$, lowered $\mathrm{mI} / \mathrm{Cr}$ in anaplastic astrocytoma $0.33 \pm 0.16$, and GBM $0.15 \pm 0.12$ compared with control subjects $0.49 \pm 0.07$.
Metwally et al. [16], in a study to determine the diagnostic accuracy of $\mathrm{MI} / \mathrm{Cr}$ ratio in the grading of gliomas, also found that the level of $\mathrm{MI} / \mathrm{Cr}$ was higher $(2 \pm 1.5)$ in low-grade astrocytomas than anaplastic astrocytomas. They reported that the $\mathrm{MI} / \mathrm{Cr}$ ratio had $100 \%$ sensitivity and $92.8 \%$ specificity for the grading of gliomas.

Approximately $60 \%$ of all pediatric tumors arise from the posterior fossa. In most cases, these tumors are grade IV medulloblastoma, grade I pilocytic astrocytoma, or grade II or III ependymoma [3]. Sometimes, a cystic/ necrotic medulloblastoma may have similar imaging features to posterior fossa pilocytic astrocytoma. Furthermore, it is often difficult to distinguish medulloblastoma from ependymoma due to similar appearance on conventional imaging. MRS and diffusion imaging are particularly useful for the differentiation of these lesions [3].

The two cases in this study that were histologically proven to be medulloblastomas, showed higher levels of choline than the other posterior fossa tumors with slight increase of myo-inositol, low NAA levels, lactate peaks, and no lipids. Mittal [18] described elevated choline and taurine peak with dwarfing of other metabolites and no lipid or lactate peak. This was in agreement with the results of this study apart from the taurine peak which is detected in only $60 \%$ of medulloblastomas.

Majos et al. [19] in a comparative study of medulloblastoma to low-grade glioma found three to fourfold increase in Cho level with markedly decreased NAA compared with low-grade gliomas. In the study done by Cuellar-Baena et al. [20], they also found that medulloblastomas had higher choline levels than ependymomas and pilocytic astrocytomas with lower NAA levels and lactate peaks. They explained this metabolic profile to be due to increased membrane turnover, low neuronal viability, and glycolysis alterations.

Ependymomas, however, had higher myo-inositol than medulloblastoma or pilocytic astrocytoma. They also had relatively high choline levels, particularly grade III ependymoma, but falling between medulloblastoma and pilocytic astrocytoma. Both ependymomas and medulloblastomas had lactate peaks with no lipids. However, Yuh et al. [21] reported that there is usually choline elevation with reduction of NAA in ependymoma, so there is no significant difference in-between ependymoma and medulloblastoma due to variability of Cho/NAA within each tumor.

In this study, pilocytic astrocytomas had mildly increased Cho and $\mathrm{Cho} / \mathrm{Cr}$ ratio with low levels of $\mathrm{Cr}$ and $\mathrm{mI}$ which is consistent with the low cellularity of the tumor. They also showed elevated lactate doublet and no lipid peak. Panigrahy et al. [3] also found that the $\mathrm{Cr}$ level and Lipids are characteristically low in pilocytic astrocytoma, but mean lactate levels are higher than in other tumors.

Cecil et al. [22] stated that pilocytic astrocytomas exhibit elevated lactate and Cho and diminished levels of NAA, Cr, 
and $\mathrm{mI}$. Hwang et al. [23] suggested that the high lactate level in the benign tumor may be due to an abnormal number or dysfunction of mitochondria, which would interfere with the process of oxidative phosphorylation and electron transport, alterations in proportional oxygen delivery, and oxygen extraction or usage by tumor or anaerobic glycolysis by tumor cells.

Diffuse pontine gliomas account for $10-15 \%$ of childhood brain tumors [24]. There were two cases of diffuse pontine gliomas in this study diagnosed radiologically and by clinical follow-up. They showed elevation of choline and reduced NAA and creatine thus confirming neoplastic nature. They also showed relatively low $\mathrm{Cho} / \mathrm{Cr}$ and $\mathrm{Cho} / \mathrm{NAA}$ ratios with no lipid or lactate peaks.

To differentiate primary high-grade glioma from solitary metastases, Cho level is measured outside the tumor margin or enhancing edge. In the current study, the Cho/NAA and $\mathrm{Cho} / \mathrm{Cr}$ ratios measured outside the margin of high-grade gliomas ranged from 1.7 to 7 and from 2.4 to 3.5 respectively while in the case of metastasis, they measured 0.5 and 0.7 . This was in agreement with the results of Al-Okaili et al. [1] who used a perilesional Cho/NAA ratio of 1 outside the enhanced or T2 defined margin. This showed $100 \%$ accuracy in discrimination of primary from secondary neoplasms. Tsougos et al. [25] found that the $\mathrm{Cho} / \mathrm{Cr}$ ratio measured in the peritumoral region had $89 \%$ sensitivity and $62 \%$ specificity at a cutoff value 1.4, while the Cho/NAA showed $78 \%$ sensitivity and $93 \%$ specificity at a cutoff value 1.1.

A limitation of this study is the inclusion of a small number of cases. Further studies are needed to quantify the extent to which MRS facilitates diagnosis, changes pediatric patient management, and its impact on the outcome of these patients.

\section{Conclusion}

MRS brings us a step closer to making a confident noninvasive diagnosis of pediatric brain tumors in clinical practice particularly in cases where biopsy could be associated with significant morbidity. The use of certain metabolite ratios with high sensitivity and specificity to distinguish neoplastic from non-neoplastic lesions as well as low-grade from high-grade neoplasms can potentially improve outcomes through avoiding biopsy of indolent lesions and facilitating earlier treatment planning.

\section{Abbreviations \\ Cho: Choline; Cr: Creatine; MI: Myo-inositol; MRI: Magnetic resonance imaging; MRS: Magnetic resonance spectroscopy; NAA: N-acetylaspartate}

\section{Acknowledgements}

Not applicable.

\section{Authors' contributions}

All authors contributed in the work presented in this manuscript. KR performed the clinical examinations, collected the clinical and histopathological data for all the patients, and referred the patients to the Radiology Department in Assiut University Hospital for MRI and MRS examination. NA and GK supervised the MRI and MRS examination. SS, NA, and GK interpreted the images, prepared the patients' reports, and analyzed the data. All the authors participated in the writing of this manuscript then it was read and approved by all authors.

\section{Funding}

This work was not supported by any grants or companies or institutional funds.

\section{Availability of data and materials}

The datasets used and/or analyzed during the current study are available from the corresponding author on reasonable request.

\section{Ethics approval and consent to participate}

As the patients were less than 16 years old, informed written consents were obtained from the patients' guardians before enrolment according to the National Ethics Committee.

This study was approved by the Local Ethics Committee of the Faculty of Medicine, Assiut University.

Committee's reference number is not applicable.

\section{Consent for publication}

As the patients were less than 16 years old when consent for publication was requested, written informed consent for the publication of this data was given by their parent or legal guardian.

\section{Competing interests}

The authors declare that they have no competing interests.

\section{Author details}

${ }^{1}$ Radiology Department, Faculty of Medicine, Assiut University Hospitals, Assiut University, Assiut 71526, Egypt. ${ }^{2}$ Pediatric Oncology Department, South Egypt Cancer Institute, Assiut 71515, Egypt.

Received: 24 October 2019 Accepted: 8 January 2020

Published online: 15 January 2020

\section{References}

1. Al-Okaili RNKJ, Woo JH, Wolf RL, O'Rourke DM, Judy KD et al (2007) Intra axial brain masses: MR imaging-based diagnostic strategy--initial experience. Radiology 243(2):533-550

2. Al-Okaili RNKJ, Krejza J, Wang S, Woo JH, Melhem ER et al (2006) Advanced MR imaging techniques in the diagnosis of intra axial brain tumors. RadioGraphics 26:S173-S189

3. Panigrahy A, Nelson MD Jr, Blüml S (2010) Magnetic resonance spectroscopy in pediatric neuroradiology: clinical and research applications. Pediatr Radiol 40:3-30

4. Zarifil M, Tzika AA (2016) Proton MRS imaging in pediatric brain tumors. Pediatr Radiol 46:952-962

5. Shiroishi MS, Panigrahy A, Moore KR, Nelson MD Jr, Gilles FH, Gonzalez-Gomez l et al (2015) Combined MRI and MRS improves pre-therapeutic diagnoses of pediatric brain tumors over MRI alone. Neuroradiology 57(9):951-956

6. Manias K, Gill SK, Zarinabad N, Davies P, English M, Ford D et al (2018) Evaluation of the added value of $1 \mathrm{H}$-magnetic resonance spectroscopy for the diagnosis of pediatric brain lesions in clinical practice. Neuro-Oncology Practice 5(1):18-27

7. Young GS (2007) Advanced MRI of adult brain tumors. Neurol Clin 25:947-937

8. Luthra G, Parihar A, Nath K, Jaiswal S, Prasad KN, Husain N et al (2006) Comparative evaluation of fungal, tubercular, and pyogenic brain abscesses with conventional and diffusion MR imaging and proton MR spectroscopy. Am J Neuroradiol 28:1332-1338

9. Spampinato MV, Smith JK, Kwock L, Ewend M, Grimme JD, Camacho DL, Castillo M (2007) Cerebral blood volume measurements and proton MR spectroscopy in grading of oligodendroglial tumors. AJR Am J Roentgenol 188(1):204-212

10. Karatag O, Karatag GY, Uysal E, Meltem Can S, Erturk M, Basak M (2010) Can magnetic resonance spectroscopy adequately differentiate neoplastic from non-neoplastic and high grade from low grade lesions in brain masses? Marmara Med J 23(3):326-338

11. McKnight TR, Lamborn KR, Love TD, Berger MS, Chang S, Dillon WP et al (2007) Correlation of magnetic resonance spectroscopic and growth characteristics within grades II and III gliomas. J Neurosurg 106:660-666 
12. Butzen J, Prost R, Chetty V, Donahue K, Neppel R, Bowen W et al (2000) Discrimination of neoplastic and non-neoplastic brain lesions by use of proton MR spectroscopy; the limit of accuracy with a logistic registration model. Am J Neuro Radiol 21(7):1213-1219

13. Bulik M, Jancalek R, Vanicek J, Skoch A, Mechl M (2013) Potential of MR spectroscopy for assessment of glioma grading. Clin Neurol Neurosurg 115:146-153

14. Kapsalaki E, Gotsis ED, Fountas KN (2008) The role of proton magnetic resonance spectroscopy in the diagnosis and categorization of cerebral abscesses. Neurosurg Focus 24(6):166-177

15. Oz G, Alger JR, Barker PB, Bartha R, Bizzi A, Boesch C et al (2014) Clinical proton MR spectroscopy in central nervous system disorders. Radiology 270(3):658-679

16. Metwally LIA, Emad El-din S, Abdelaziz O, Hamdy IM, Elsamman AK, Abdelalim AM (2014) Predicting grade of cerebral gliomas using myoinositol/creatine ratio. Egypt J Radiol Nuclear Med 45:211-217

17. Castillo SJ, Smith KJ, Kwock L (2000) Correlation of myo-inositol levels and grading of cerebral astrocytomas. Am J Neuro Radiol 21(9):1645-1649

18. Mittal P (2011) Magnetic resonance spectroscopy findings in non-enhancing desmoplastic medulloblastoma. Ann Indian Acad Neuro 14(3):200-202

19. Majos C, Alonso J, Aguilera C, Serrallonga M, Acebes JJ, Arus C et al (2002) Proton MR spectroscopic findings with possible application for differential diagnosis. Radiology 556:225-266

20. Cuellar-Baena S, Morales JM, Martinetto H, Calvar J, Sevlever G, Castellano G et al (2010) Comparative metabolic profiling of paediatric ependymoma, medulloblastoma and pilocytic astrocytoma. Int J Mol Med 26:941-948

21. Yuh EL, Barkovich AJ, Gupta N (2009) Imaging of ependymoma. MRI and CT Childs Nerv System 25(10):1203-1213

22. Cecil KM, Jones BV (2001) Magnetic resonance spectroscopy of the pediatric brain. Top Magn Reson Imaging 12(6):435-452

23. Hwang JH, Egnaczyk GF, Ballard E, Dunn RS, Holland SK, Ball WS Jr (1998) Proton MR spectroscopic characteristics of pediatric pilocytic astrocytomas. AJNR Am J Neuroradiol 19(3):535-540

24. Yamasaki F, Kurisu K, Kajiwara Y, Watanabe Y, Takayasu T, Akiyama Y et al (2011) Magnetic resonance spectroscopic detection of lactate is predictive of a poor prognosis in patients with diffuse intrinsic pontine glioma. NeuroOncology 13(7):791-801

25. Tsougos I, Svolos P, Kousi E, Fountas K, Theodorou K, Fezoulidis I et al (2012) Differentiation of glioblastoma multiforme from metastatic brain tumor using proton magnetic resonance spectroscopy, diffusion and perfusion metrics at 3T. Cancer Imaging 17:423-436

\section{Publisher's Note}

Springer Nature remains neutral with regard to jurisdictional claims in published maps and institutional affiliations.

\section{Submit your manuscript to a SpringerOpen ${ }^{\circ}$ journal and benefit from:}

- Convenient online submission

- Rigorous peer review

- Open access: articles freely available online

- High visibility within the field

- Retaining the copyright to your article

Submit your next manuscript at $\boldsymbol{\nabla}$ springeropen.com 